\title{
Major unsolved points in adult neurogenesis: doors open on a translational future?
}

\author{
Paolo Peretto $^{1,2 *}$ and Luca Bonfanti ${ }^{1,3 *}$ \\ ${ }^{1}$ Neuroscience Institute Cavalieri Ottolenghi, Orbassano, Italy \\ 2 Department of Life Sciences and System Biology, University of Turin, Torino, Italy \\ ${ }^{3}$ Department of Veterinary Sciences, University of Turin, Torino, Italy
}

Edited by:

Verdon Taylor, University of

Sheffield, UK

\section{Reviewed by:}

Hans Georg Kuhn, University of

Gothenburg, Sweden

Nicolas Toni, University of

Lausanne, Switzerland

\section{*Correspondence:}

Paolo Peretto, Department of Life

Sciences and Systems Biology,

University of Turin, Via Accademia

Albertina 13, 10123 Torino, Italy

e-mail: paolo.peretto@unito.it;

Luca Bonfanti, Department of

Veterinary Sciences, University of

Turin, Via Leonardo da Vinci, 44,

10095 Grugliasco, Italy

e-mail: luca.bonfanti@unito.it
In spite of many data gathered during the last two decades on adult neurogenesis (AN) it is evident that such knowledge is not sufficient for granting translational outcomes in brain repair, especially if the ultimate goal is to promote cell replacement. Alternative strategies aimed at fostering AN physiological functions (restorative approaches) are still undefined. By asking the question whether AN research field has to be considered as a dead end in the context of brain repair, here we review some unresolved issues: multifaceted evolutionary constraints in mammals, stem/progenitor cell type/availability and tissue permissivity, impact on other brain functions, interplay with other forms of plasticity, and relevance in humans. We suggest that full understanding of AN biology is an essential step for its possible exploitation in brain repair, and that further fundamental, multidisciplinary research is required to reach translational outcomes. Scientist's attitude and their communication skills are also important. To avoid overestimation of AN reparative potential in a translational perspective, more distant goals of cell replacement should be kept clearly distinct from restorative approaches involving AN functional plasticity.

Keywords: brain repair, neurodegenerative diseases, regenerative medicine, therapeutic approaches, neural stem cells, parenchymal progenitors, cell therapy, brain evolution
Two decades of investigation on adult neurogenesis (AN) yielded an utterly new vision of brain plasticity and opened new perspectives for brain repair/regeneration strategies. Nevertheless, the ultimate goal of exploiting neurogenic processes for obtaining cell replacement is still far from being achieved. Starting from this antinomy, the big question is: should be the AN research field considered as a dead end in the perspective of brain repair, or, alternatively, is it worthwhile to put in place further efforts in order to solve the problem? By reading the scientific literature, it is clear that all neurobiologists, even believing in an AN translational future, do not share the same answer. Non-univocal visions are normal in a field that has developed by progressively ramifying in many directions accordingly to the different goals pursued by each research group. Some scientists primarily deal with AN physiological roles/mechanisms, apparently being less interested in direct translation of results. Others are mainly focused on aspects that implement $\mathrm{AN}$, or directly address the issue of injury-induced, reactive neurogenesis, paying less attention to the peculiar limits of the mammalian CNS in repairing damage. New translational perspectives in "restorative" rather than "structural reparative" neurology have been recently raised, what could be useful in slowing down the impact of various neurologic impairments (e.g., those occurring in neurodegenerative, vascular, traumatic diseases, age-related cognitive decline), even in the absence of cell replacement. Nevertheless, it is evident that knowledge gathered during the last two decades is not yet sufficient for granting translation of basic neurobiological research. Such inability is linked to several unresolved issues in both physiological and lesion-induced neurogenesis, and to scarcely integrated views between different approaches used to address AN studies. In other words, even in the absence of current, effective therapeutic outcomes, we may not be at a dead end, rather we are in the middle of a route with many new "perfectly reasonable deviations from the beaten track" (Feynman, 2005).

\section{THE PRESENT KNOWLEDGE IN MAMMALS: SOME LIGHTS IN THE DARK}

Our knowledge of AN in mammals might be grouped in two domains: first, some facts and concepts which are definitively accepted and substantially understood by the scientific community ("acquired knowledge"), and second, a number of issues which remain largely obscure and/or underestimated ("gaps of knowledge"). The main blocks of acquired knowledge can be summarized as follows: (i) two canonical neurogenic zones (subventricular zone, SVZ, and subgranular zone, SGZ) harboring stem cell niches provide neural cell addition into the olfactory bulb and hippocampus (Ming and Song, 2011); we know a lot about their anatomical organization and functional regulation as well as the integration of the newly born neurons (Fuentealba et al., 2012; Tong and Alvarez-Buylla, 2014; Vadodaria and Gage, 2014); (ii) wide areas of the central nervous system (CNS) out of the canonical neurogenic sites host cycling and/or quiescent progenitors which give rise to different processes of non-canonical cell genesis: parenchymal gliogenesis (Boda and Buffo, 2010; Trotter et al., 2010), parenchymal neurogenesis (Bonfanti and Peretto, 2011) and periventricular neurogenesis (Migaud et al., 
2010); little is known about non-canonical cell genesis, which seems to lack integration within the parenchyma; (iii) progenitors in both canonical and non-canonical neurogenic sites are activated in different pathological conditions (Arvidsson et al., 2002; Luzzati et al., 2011); in spite of such activation, the response to injury is substantially non-coordinated and/or abortive, not leading to effective brain repair (Kernie and Parent, 2010; Bonfanti, 2011; Bonfanti and Peretto, 2011).

Behind these blocks of acquired knowledge large amounts of unknown facts and concepts are still hidden. First of all, AN remarkable plasticity has introduced a new kind of complexity: that of dynamic, developmental-like processes related to neuronal addition occurring within a substantially static tissue. Moreover, in mammals, the CNS is structurally, functionally, and evolutionarily refractory to repair, healing, and regeneration. These facts make it extremely challenging to exploit AN as a therapeutic tool, also because the variables involved are dependent among each other and linked by different hierarchies (Figure 1). Here, we will analyze the key points still remaining open in the AN field, considering them as potential hurdles to a full understanding of the biological process itself, and, in turn, to its possible exploitation for brain repair.

\section{AN PHYSIOLOGICAL FUNCTION(S) vs. BRAIN REPAIR: EVOLUTIONARY ASPECTS}

Unlike most vertebrates, in adult mammals spontaneous neurogenesis is primarily linked to homeostatic/physiological roles and hardly directed to repair (Bonfanti, 2011). This view is supported by many studies which addressed the issue of reactive (lesion-induced) neurogenesis, indicating "activation" of neural progenitors which substantially do not provide cell replacement, most of the newly born/mobilized cells being fated to die (Kernie and Parent, 2010; Luzzati et al., 2011).

The fact that many non-mammalian vertebrates can perform brain repair/regeneration (Endo et al., 2007; Grandel and Brand, 2013) underlines the involvement of evolutionary aspects at the developmental, anatomical, stem cell types/availability and tissuespecific environment levels (Bonfanti, 2011). The occurrence of AN in the CNS of all vertebrates suggests the naive (and wrong) view of a direct relationship between neurogenic activity and regenerative capability (Ferretti, 2011): AN is not sufficient for regeneration to occur, and other aspects should be considered. Beside lower intrinsic regenerative properties (and lower availability of stem/progenitor cells), the mammalian CNS is characterized by more detrimental tissue reactions, in fact hampering regeneration. Immune cell activation leading to inflammation is an early response after injury that is common to most animal groups. Yet, whereas in many non-mammalian vertebrates initial acute inflammation stimulates regeneration without subsequent detrimental tissue responses, in mammals neuroinflammation leads to the formation of the glial scar with consequent impairment of regeneration (Mescher and Neff, 2006; Sofroniew, 2009; Kyritsis et al., 2014). In other words, stem/progenitor cell availability alone cannot grant regenerative capacity if glial cell activation and inflammatory reactions also occur. A theory explains the failure in mammalian brain repair as a result of evolutionary constraints in which the injured CNS would not favor a strategy of regeneration, but rather one of minimizing further damage (Weil et al., 2008). Hence, important gaps of knowledge still exist, both in mammals and other vertebrates, concerning homeostastic/metabolic functions and tissue reaction aspects linked to $\mathrm{AN}$, and the role of the immune system, which still remain largely unexplored (Schwartz et al., 2013). All these variables are involved in determining the differences between neurogenic and non-neurogenic tissue local environments, and, in turn, their permissivity to reparative processes.

\section{PROGENITOR CELLS, TISSUE ENVIRONMENT, AND AN OUTCOME(S)}

Although cell proliferation exists throughout the intact CNS and is enhanced by several physiological/pathological conditions, it does not produce substantial neurogenic outcomes in the parenchyma out of the canonical sites (olfactory bulb, dentate gyrus). The main aspects that seem essential in granting CNS neurogenic/reparative capacity are: occurrence of specialized progenitor types and tissue permissivity. The SVZ and SGZ neurogenic niches harbor stem cells that appear very specified in their commitment (Obernier et al., 2014) and thus hard to divert toward other fates. As to parenchymal neural progenitors in non-canonical sites it is not yet clear what is their origin, nature, fate, and function(s). Yet, these cells do represent promising substrates for future research for several reasons (abundance, widespread distribution, region-specific differentiative commitment). Wherever stem/progenitor cells are located, both in canonical and non-canonical sites, unraveling the mechanisms underlying their quiescence or activation is also essential for their possible manipulation (Basak et al., 2012). Nevertheless, the functional availability of proper stem/progenitor cells is not sufficient to grant $\mathrm{AN}$ and repair in the absence of a receptive tissue environment: olfactory bulb and hippocampus circuits are permissive to neuronal integration, whereas the mature parenchyma allows less or no integration (Bonfanti and Peretto, 2011).

Taken together, these facts add hurdles to the ultimate goal of making mammalian AN processes useful for cell replacement. In spite of a large amount of data concerning the regulation of canonical AN (in terms of modulation; see Kempermann, 2011), very little is known about the cellular/molecular factors which allow the interaction between progenitors and the mature CNS tissue (permissivity) both in neurogenic and non-neurogenic sites. Such tissue permissivity is strictly linked to intrinsic features (adhesion molecules, extracellular matrix, availability of growth factors, etc.) which are maintained and/or delayed from development, thus allowing the AN process to persist during adulthood. In this context, a few studies have thoroughly investigated the steps that drive and regulate the shift between embryonic and AN.

\section{ANIMAL MODELS vs. HUMANS}

Most knowledge on AN has been gathered on laboratory rodents, what is a clear limit for translational approaches. Indeed, neurogenic processes differ quite among mammals as to their location, rate, niche organization, and the postnatal temporal windows in which they occur (Bonfanti and Peretto, 2011). For instance, 


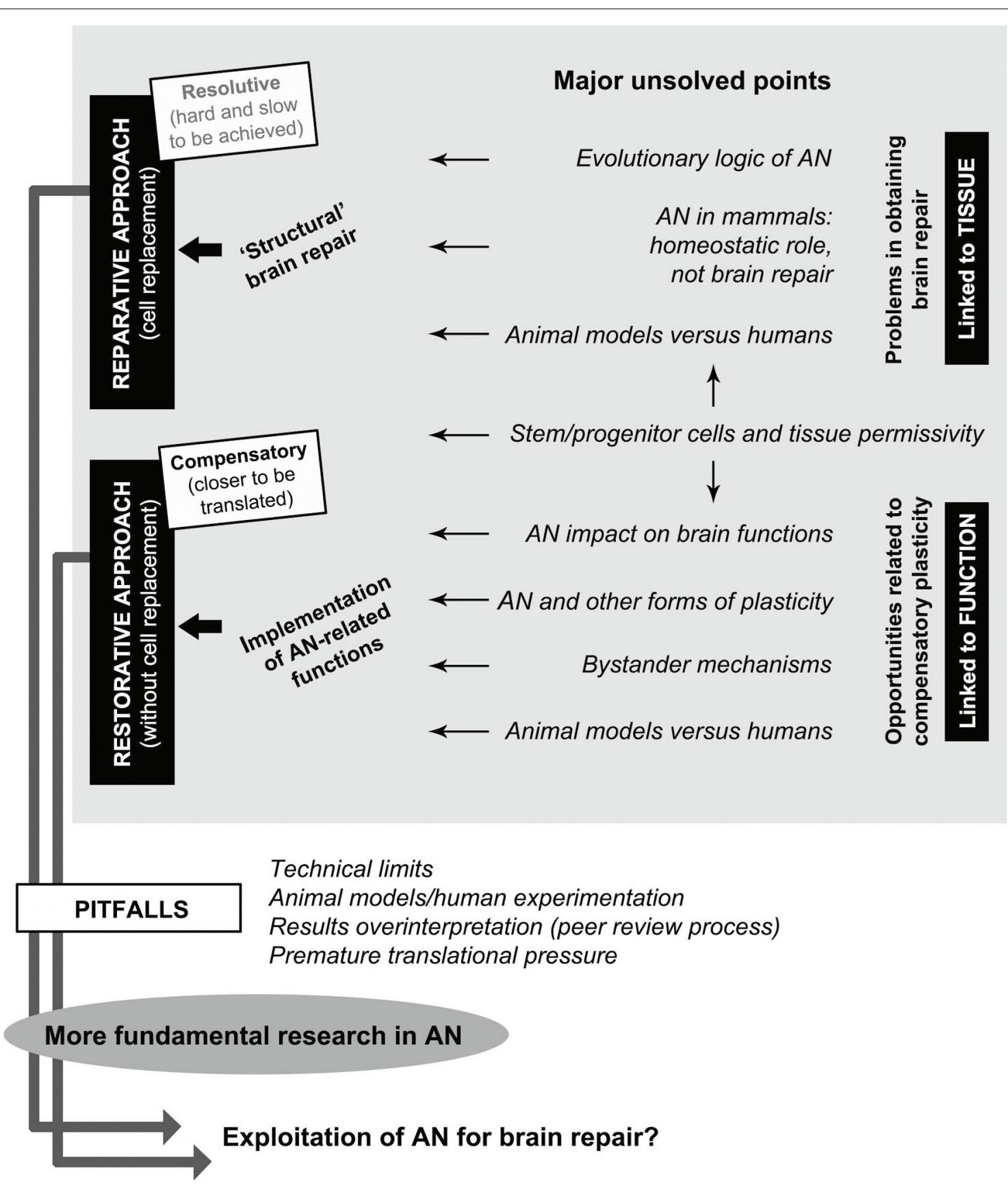

FIGURE 1 | Interplay between adult neurogenesis (AN), some of its major unsolved points, and possible perspectives for brain repair. Gray box: among many open issues still existing in the potential role of $A N$ in neural plasticity (gaps of knowledge), a general distinction should be made between: (i) tissue-related problems depending on evolutionary issues and hampering brain repair/regeneration (top), and (ii) function-related opportunities depending on possible homeostatic roles of $\mathrm{AN}$ which could be exploited/implemented for restorative approaches (bottom).

Stem/progenitor cell availability and tissue permissivity (middle) are essential aspects for allowing translational perspectives to be figured out in both directions. Reparative approaches, which imply cell replacement as the ultimate goal of regenerative medicine, are not available at present. Restorative approaches include different therapeutic perspectives linked to the implementation of physiological functions of AN aimed at obtaining compensatory plasticity in the absence of cell replacement, both in the damaged and undamaged (age-related decline) brain. Successful achievement of these goals is linked to further investments in fundamental research by overcoming of current pitfalls in the AN field. while cell migration from SVZ to olfactory bulb persists throughout life in mice, it is exhausted very early in human infants (Sanai et al., 2011). In the human hippocampus, measures of ${ }^{14} \mathrm{C}$ concentration in genomic DNA show a substantially constant rate of AN through ages, in contrast with an evident decrease in young rodents (Spalding et al., 2013). Also parenchymal neurogenesis remarkably varies among mammals, showing species-specific regional localizations (Luzzati et al., 2006; Ponti et al., 2008). 
Other inter-mammalian differences concern specific functions related to the ecological needs and behavioral activity of the animals (Barker et al., 2011).

Besides AN heterogeneity, mammals also differ in their brain anatomy and physiology (Carlson, 2012), and this can affect the impact AN might exert on the whole brain function (see below). Also the average lifespan varies among mammals, thus implying that differences in postnatal development of CNS areas, brain maturation, puberty, make it difficult to compare AN in different species (Lindsey and Tropepe, 2006; Kuhn and Blomgren, 2011). Hence, restraining AN research to laboratory rodents may introduce several bias in the search for translational outcomes. If comparative/evolutionary studies through phylogeny are essential to unravel the common logic of $\mathrm{AN}$, the study of differences among mammals are also important for correctly interpreting/modeling the possible contribution of $\mathrm{AN}$ to homeostasis and brain repair in humans.

\section{TO WHICH EXTENT AN IMPACTS THE BRAIN FUNCTION?}

Addition of newborn neurons in the olfactory bulb and hippocampus optimizes neurological functions/behaviors such as social interaction/reproduction, memory, learning, and pattern separation (Sahay et al., 2011; Feierstein, 2012). These two brain regions are essential for behavioral outputs critical for survival of the individuals and species (MucignatCaretta et al., 2012; Snyder and Cameron, 2012). Accordingly, $\mathrm{AN}$ is assumed as a mechanism which promotes life-long adaptability of individuals to environmental complexity and novelty (Freund et al., 2013). Regulation of AN is achieved through integration of multiple external and internal stimuli, which implies activity of multiple brain regions/circuits and complex feedback loops (Kempermann, 2011). Thus, though restricted to the olfactory bulb and hippocampus, AN potentially impacts diverse brain functions (Snyder and Cameron, 2012; Lepousez et al., 2013), which might explain that in mammals it occurs only in two regions. Although little is known on this hypothesis, since the anatomical, functional, molecular bases underlying the above mentioned interactions are far from being clarified, the possible impact of AN on other brain functions/circuits can have important translational implications (Leuner and Gould, 2010; Kheirbek et al., 2012; Snyder and Cameron, 2012; Quadrato et al., 2014). Several data are already available on the link between hippocampus, pattern separation/overgeneralization of sensory stimuli and anxiety disorders (Leuner and Gould, 2010; Kheirbek et al., 2012). The recent finding that human hippocampal AN appears substantially maintained during adulthood (Spalding et al., 2013) adds new interest to this issue, also in the perspective of implementing cognitive functions during aging (Bordey, 2014). Yet, proper translational outcomes imply definitive clarification of the real rate/impact of $\mathrm{AN}$ in humans during postnatal development and adulthood, in physiological, and pathological condition.

Finally, if AN does extensively affect the brain function(s), it should be emphasized that it is only one among other forms of CNS plasticity and that very little is known about the mechanisms which underlie their mutual relationships.

\section{AN AND OTHER FORMS OF PLASTICITY AND/OR REPAIR STRATEGIES}

The CNS of mammals, in spite of having lost most of its regenerative/repair capacity with respect to other phyla, is endowed with different forms of structural plasticity involving pre-existing cellular elements (Bonfanti and Nacher, 2012). Among them, the most known and widespread is the experience-dependent synaptic plasticity that can occur in response to environmental enrichment and after a lesion in the form of compensatory events, i.e., synaptic formation/elimination and axonal sprouting/pruning (Brown et al., 2009; Chen and Nedivi, 2010; Fu and Zuo, 2011). Further levels of structural plasticity are found in a population of "immature," non-newly generated neurons of the cerebral cortex (Gomez-Climent et al., 2008). These cells, in spite of their differentiated neuronal morphology (Luzzati et al., 2009), express immature neuronal markers and show very few synapses on their membrane, thus not being integrated in the adult cortical circuits, like "stand by" elements (Bonfanti and Nacher, 2012). All these forms of structural plasticity could be useful in rehabilitation approaches that mostly exploit compensatory plasticity of undamaged, preexisting structures (Dobkin, 2004). If and how all these forms of plasticity are integrated with AN is a fully open question, also taking into account that mammalian AN itself consists of heterogeneous processes involving the canonical neurogenic niches and progenitors located throughout the CNS tissue (non-canonical cell genesis; Boda and Buffo, 2010; Bonfanti and Peretto, 2011).

A better knowledge of the mutual relationships existing within the vast landscape of neural plasticity is fundamental to correctly figure out restorative therapeutic approaches in neurology (Figure 1). In recent years, several studies have started to unravel new modes of communication between stem/progenitor cells (endogenous or transplanted) and resident cells of the CNS, also involving a cross-talk with the immune system. This communication is at the basis of the so called "bystander effects," namely a series of paracrine mechanisms which can exert beneficial effects even in the absence of cell replacement (Martino et al., 2011). An hypothesis supported by several works is that transplanted stem/progenitor cells can exert a bystander immune modulation by modifying the inhospitable microenvironment at the injury site through the release of soluble molecules such as chemokines and cytokines (Pluchino and Cossetti, 2013). More recently, it has been proposed that the same effects might be also exerted by cell mobilization/activation of endogenous stem/progenitor cells toward adjacent injured sites (Kokaia et al., 2012). In perspective, these studies have the added value of considering neural plasticity, AN, and brain repair in the context of a cross-talk between the CNS and the immune system, the latter being far more important than previously thought. Hence, the study of cell-cell interaction/paracrine communication does represent a fully open, promising field of research, aimed at developing "restorative" rather than "cell replacement" strategies.

\section{SOME PITFALLS IN AN FIELD: INTERPRETATION OF RESULTS AND PEER REVIEW PROCESS}

Since the beginning, following the emphasis of a new form of CNS plasticity, the reparative potential of AN has been overestimated 
by the scientific community, at least under its possible regenerative outcome. This fact is reflected by statements contained in many papers dealing with both spontaneous and lesion-induced AN in which the results obtained are more or less directly linked with potential therapeutic outcomes, in the absence of direct evidence for such a link. These statements, although originally intended as "possibilities" by the authors, are frequently amplified by the media, thus generating unjustified hopes in patients affected by neurological diseases (this aspect is analyzed in Cattaneo and Bonfanti, 2014). The source of this problem is well addressed by Kerner (2006): "Many individual research reports, while suggesting exciting new innovations that may lie ahead in the future, have little or no immediate application in public health and/or clinical practice. Thus, it may be difficult for the practice community to distinguish the signal about what is currently important to practice from the noise of what may or may not become important in the future."

In the neurological context, restorative approaches in the absence of cell replacement, including modulation of physiological/paracrine functions (Martino et al., 2011; Bordey, 2014; Quadrato et al., 2014) should always be kept clearly distinct from the true reparative/regenerative processes involving cell replacement. In the history of $\mathrm{AN}$ scientific publications, from the initial "naïve" belief that AN could easily represent the biological substrate for cell replacement in the CNS, to a more recent overestimation of the bystander effect therapeutic potential, it appears that too many unjustified claims actually bypass the filter of peer review. We feel that this habit is not rewarding for the public representation of science and even not for the future of AN field.

\section{FUTURE PERSPECTIVES: AN AND FUNDAMENTAL RESEARCH}

It appears evident that having introduced excessive and premature translational issues toward brain repair did not solve the problem of neurological diseases. Moreover, a simplistic view of $\mathrm{AN}$ as a ready-made therapeutic tool could even be counterproductive, since it might put down the interest in AN studies. In spite of some oversights along the route, the increasing knowledge gathered during the last 20 years is enormous, considering the changes that $\mathrm{AN}$ research has produced in our vision of brain plasticity. Non-invasive technologies are essential to study $\mathrm{AN}$ in humans (e.g., Spalding et al., 2013 for ${ }^{14} \mathrm{C}$ detection), although further technical advancements are needed (e.g., cell imaging enhanced resolution; Sierra et al., 2011). Yet, because the AN field has become widely diversified both in its goals and lines of research (Figure 1), obtaining of future breakthroughs will require a multidisciplinary integration of high specific expertise in order to gain a whole transversal view of the variables involved (for the several meanings of translation/implementation concepts see Kerner, 2006). The current gaps of knowledge should be filled with new basic research prior to put them into a translational view. We can be confident that applied (beneficial and profitable) products of fundamental research will be eventually achieved in the future, although not visible/predictable at the time of the experimental phases (Press, 2013). Confidence in the eventual usefulness of basic research should be sufficient to bring back $\mathrm{AN}$ in the normal context of science.

\section{ACKNOWLEDGMENT}

We thank Carlos Lois for reading the manuscript and for his precious advices.

\section{REFERENCES}

Arvidsson, A., Collin, T., Kirik, D., Kokaia, Z., and Lindvall, O. (2002). Neuronal replacement from endogenous precursors in the adult brain after stroke. Nat. Med. 8, 963-970. doi: 10.1038/nm747

Barker, J. M., Boonstra, R., and Wojtowicz, J. M. (2011). From pattern to pourpose: how comparative studies contribute to understanding the function of adult neurogenesis. Eur. J. Neurosci. 34, 963-977. doi: 10.1111/j.1460-9568.2011. 07823.x

Basak, O., Giachino, C., Fiorini, E., Macdonald, H. R., and Taylor, V. (2012). Neurogenic subventricular zone stem/progenitor cells are Notch1-dependent in their active but not quiescent state. J. Neurosci. 32, 5654-5666. doi: 10.1523/JNEUROSCI.0455-12.2012

Boda, E., and Buffo, A. (2010). Glial cells in non-germinal territories: insights into their stem/progenitor properties in the intact and injured nervous tissue. Arch. Ital. Biol. 148, 119-136.

Bonfanti, L. (2011). From hydra regeneration to human brain structuralplasticity: a long trip through narrowing roads. Sci.World J. 11, 1270-1299. doi: 10.1100/tsw.2011.113

Bonfanti, L., and Nacher, J. (2012). New scenarios for neuronal structural plasticity in non-neurogenic brain parenchyma: the case of cortical layer II immature neurons. Prog. Neurobiol. 98, 1-15. doi: 10.1016/j.pneurobio.2012.05.002

Bonfanti, L., and Peretto, P. (2011). Adult neurogenesis in mammals-a theme with many variations. Eur. J. Neurosci. 34, 930-950. doi: 10.1111/j.14609568.2011.07832.x

Bordey, A. (2014). Endogenous stem cells for enhancing cognition in the diseased brain. Front. Neurosci. 8:98. doi: 10.3389/fnins.2014.00098

Brown, C. E., Aminoltejari, K., Erb, H., Winship, I. R., and Murphy, T. H. (2009). In vivo voltage-sensitive dye imaging in adult mice reveals that somatosensory maps lost to stroke are replaced over weeks by new structural and functional circuits with prolonged modes of activation within both the peri-infarct zone and distant sites. J. Neurosci. 29, 1719-1734. doi: 10.1523/JNEUROSCI.424908.2009

Carlson, B. A. (2012). Diversity matters: the importance of comparative studies and the potential for synergy between neuroscience and evolutionary biology. Arch. Neurol. 69, 987-993. doi: 10.1001/archneurol.2012.77

Cattaneo, E., and Bonfanti, L. (2014). Stem cell therapeutic potential is higher in society than in the brain. Front. Neurosci. 8:79. doi: 10.3389/fnins.2014.00079

Chen, J. L., and Nedivi, E. (2010). Neuronal structural remodeling: is it all about access? Curr. Opin. Neurobiol. 20, 557-562. doi: 10.1016/j.conb.2010. 06.002

Dobkin, B. H. (2004). Neurobiology of rehabilitation. Ann. N.Y. Acad. Sci. 1038, 148-170. doi: 10.1196/annals.1315.024

Endo, T., Yoshino, J., Kado, K., and Tochinai, S. (2007). Brain regeneration in anuran amphibians. Dev. Growth Differ. 49, 121-129. doi: 10.1111/j.1440169X.2007.00914.x

Feierstein, C. E. (2012). Linking adult olfactory neurogenesis to social behavior. Front. Neurosci. 6:173. doi: 10.3389/fnins.2012.00173

Ferretti, P. (2011). Is there a relationship between adult neurogenesis and neuron generation following injury across evolution? Eur. J. Neurosci. 34, 951-962. doi: 10.1111/j.1460-9568.2011.07833.x

Feynman, R. P. (2005). Perfectly Reasonable Deviations (from the Beaten Track). New York, NY: Basic Books.

Freund, J., Brandmaier, A. M., Lewejohann, L., Kirste, I., Kritzler, M., Krüger, A., et al. (2013). Emergence of individuality in genetically identical mice. Science 340, 756-759. doi: 10.1126/science.1235294

Fu, M., and Zuo, Y. (2011). Experience-dependent structural plasticity in the cortex. Trends Neurosci. 34, 177-187. doi: 10.1016/j.tins.2011.02.001

Fuentealba, L. C., Obernier, K., and Alvarez-Buylla, A. (2012). Adult neural stem cells bridge their niche. Cell Stem Cell 10, 698-708. doi: 10.1016/j.stem.2012.05.012

Gomez-Climent, M. A., Castillo-Gomez, E., Varea, E., Guirado, R., Blasco-Ibanez, J. M., Crespo, C., et al. (2008). A population of prenatally generated cells in the rat paleocortex maintains an immature neuronal phenotype into adulthood. Cereb. Cortex 18, 2229-2240. doi: 10.1093/cercor/bhm255 
Grandel, H., and Brand, M. (2013). Comparative aspects of adult neural stem cell activity in vertebrates. Dev. Genes Evol. 223, 131-147. doi: 10.1007/s00427-0120425-5

Kempermann, G. (2011). Seven principles in the regulation of adult neurogenesis. Eur. J. Neurosci. 33, 1018-1024. doi: 10.1111/j.1460-9568.2011.07599.x

Kerner, J. F. (2006). Knowledge translation versus knowledge integration: a "funder's" perspective. J. Contin. Educ. Health Prof. 26, 72-80. doi: 10.1002/chp.53

Kernie, S. G., and Parent, J. M. (2010). Forebrain neurogenesis after focal ischemia and traumatic brain injury. Neurobiol. Dis. 37, 267-274. doi: 10.1016/j.nbd.2009.11.002

Kheirbek, M. A., Klemenhagen, K. C., Sahay, A., and Hen, R. (2012). Neurogenesis and generalization: a new approach to stratify and treat anxiety disorders. Nat. Neurosci. 15, 1613-1620. doi: 10.1038/nn.3262

Kokaia, Z., Martino, G., Schwartz, M., and Lindvall, O. (2012). Cross-talk between neural stem cells and immune cells: the key to better brain repair? Nat. Neurosci. 15, 1078-1087. doi: 10.1038/nn.3163

Kuhn, G. H., and Blomgren, K. (2011). Developmental dysregulation of adult neurogenesis. Eur. J. Neurosci. 33, 1115-1122. doi: 10.1111/j.14609568.2011.07610.x

Kyritsis, N., Kizil, C., and Brand, M. (2014). Neuroinflammation and central nervous system regeneration in vertebrates. Trends Cell Biol. 24, 128-135. doi: 10.1016/j.tcb.2013.08.004

Lepousez, G., Valley, M. T., and Lledo, P. M. (2013). The impact of adult neurogenesis on olfactory bulb circuits and computations. Annu. Rev. Physiol. 75, 339-363. doi: 10.1146/annurev-physiol-030212-183731

Leuner, B., and Gould, E. (2010). Structural plasticity and hippocampal function. Annu. Rev. Psychol. 61, 111-140. doi: 10.1146/annurev.psych.093008.100359

Lindsey, B. W., and Tropepe, V. (2006). A comparative framework for understanding the biological principles of adult neurogenesis. Prog. Neurobiol. 80, 281-307. doi: 10.1016/j.pneurobio.2006.11.007

Luzzati, F., Bonfanti, L., Fasolo, A., and Peretto, P. (2009). DCX and PSA-NCAM expression identifies a population of neurons preferentially distributed in associative areas of different pallial derivatives and vertebrate species. Cereb. Cortex 19, 1028-1041. doi: 10.1093/cercor/bhn145

Luzzati, F., De Marchis, S., Fasolo, A., and Peretto, P. (2006). Neurogenesis in the caudate nucleus of the adult rabbit. J. Neurosci. 26, 609-621. doi: 10.1523/JNEUROSCI.4371-05.2006

Luzzati, F., De Marchis, S., Parlato, R., Gribaudo, S., Schütz, G., Fasolo, A., et al. (2011). New striatal neurons in a mouse model of progressive striatal degeneration are generated in both the subventricular zone and the striatal parenchyma. PLOS ONE 6:e25088. doi: 10.1371/journal.pone. 0025088

Martino, G. V., Pluchino, S., Bonfanti, L., and Schwartz, M. (2011). Brain regeneration in physiology and pathology: the immune signature driving therapeutic plasticity of neural stem cells. Phys. Rev. 91, 1281-1304. doi: 10.1152/physrev.00032.2010

Mescher, A. L., and Neff, A. W. (2006). Limb regeneration in amphibians: immunological considerations. ScientificWorldJournal 6, 1-11. doi: 10.1100/tsw. 2006.323

Migaud, M., Batailler, M., Segura, S., Duittoz, A., Franceschini, I., and Pillon, D. (2010). Emerging new sites for adult neurogenesis in the mammalian brain: a comparative study between the hypothalamus and the classical neurogenic zones. Eur. J. Neurosci. 32, 2042-2052. doi: 10.1111/j.1460-9568.2010. 07521.x

Ming, G. L., and Song, H. (2011). Adult neurogenesis in the mammalian brain: significant answers and significant questions. Neuron 70, 687-702. doi: 10.1016/j.neuron.2011.05.001

Mucignat-Caretta, C., Redaelli, M., and Caretta, A. (2012). One nose, one brain: contribution of the main and accessory olfactory system to chemosensation. Front. Neuroanat. 6:46. doi: 10.3389/fnana.2012.00046
Obernier, K., Tong, K. K., and Alvarez-Buylla, A. (2014). Restricted nature of adult neural stem cells: re-evaluation of their potential for brain repair. Front. Neurosci. 8:162. doi: 10.3389/fnins.2014.00162

Pluchino, S., and Cossetti, C. (2013). How stem cells speak with host immune cells in inflammatory brain diseases. Glia 61, 1379-1401. doi: 10.1002/glia.22500

Ponti, G., Peretto, P., and Bonfanti, L. (2008). Genesis of neuronal and glial progenitors in the cerebellar cortex of peripuberal and adult rabbits. PLOS ONE 3:e2366. doi: 10.1371/journal.pone.0002366

Press, W. H. (2013). What's so special about science (and how much should we spend on it?). Science 342, 817-822. doi: 10.1126/science.342.6160.817

Quadrato, G., Elnaggar, M. Y., and Di Giovanni, S. (2014). Adult neurogenesis in brain repair: cellular plasticity vs. cellular replacement. Front. Neurosci. 8:17. doi: 10.3389/fnins.2014.00017

Sahay, A., Wilson, D. A., and Hen, R. (2011). Pattern separation: a common function for new neurons in hippocampus and olfactory bulb. Neuron 70, 582-588. doi: 10.1016/j.neuron.2011.05.012

Sanai, N., Nguyen, T., Ihrie, R. A., Mirzadeh, Z., Tsai, H. H., Wong, M., et al. (2011). Corridors of migrating neurons in the human brain and their decline during infancy. Nature 478, 382-386. doi: 10.1038/nature 10487

Schwartz, M., Kipnis, J., Rivest, S., and Prat, A. (2013). How do immune cells support and shape the brain in health, disease, and aging? J. Neurosci. 33, 17587-17596. doi: 10.1523/JNEUROSCI.3241-13.2013

Sierra, A., Encinas, J. M., and Maletic-Savatic, M. (2011). Adult human neurogenesis: from microscopy to magnetic resonance imaging. Front. Neurosci. 5:47. doi: 10.3389/fnins.2011.00047

Snyder, J. S., and Cameron, H. A. (2012). Could,adult,hippocampal,neurogenesis be relevant for human behavior? Behav. Brain Res. 14, 384-390. doi: 10.1016/j.bbr.2011.06.024

Sofroniew, M. V. (2009). Molecular dissection of reactive astrogliosis and glial scar formation. Trends Neurosci. 32, 638-647. doi: 10.1016/j.tins.2009.08.002

Spalding, K. L., Bergmann, O., Alkass, K., Bernard, S., Salehpour, M., Huttner, H. B., et al. (2013). Dynamics of hippocampal neurogenesis in adult humans. Cell 153, 1219-1227. doi: 10.1016/j.cell.2013.05.002

Tong, C. K., and Alvarez-Buylla, A. (2014). SnapShot: adult neurogenesis in the V-SVZ. Neuron 81:220-220.e1. doi: 10.1016/j.neuron.2013.12.004

Trotter, J., Karram, K., and Nishiyama, A. (2010). NG2 cells: Properties, progeny and origin. Brain Res. Rev. 63, 72-82. doi: 10.1016/j.brainresrev.2009.12.006

Vadodaria, K. C., and Gage, F. H. (2014). SnapShot: adult hippocampal neurogenesis. Cell 156:1114-1114.e1. doi: 10.1016/j.cell.2014.02.029

Weil, Z. M., Norman, G. J., DeVries, A. C., and Nelson, R. J. (2008). The injured nervous system: a Darwinian perspective. Prog. Neurobiol. 86, 48-59. doi: 10.1016/j.pneurobio.2008.06.001

Conflict of Interest Statement: The authors declare that the research was conducted in the absence of any commercial or financial relationships that could be construed as a potential conflict of interest.

Received: 25 March 2014; paper pending published: 17 April 2014; accepted: 25 May 2014; published online: 11 June 2014.

Citation: Peretto P and Bonfanti L (2014) Major unsolved points in adult neurogenesis: doors open on a translational future? Front. Neurosci. 8:154. doi: 10.3389/fnins. 2014.00154

This article was submitted to Neurogenesis, a section of the journal Frontiers in Neuroscience.

Copyright (C) 2014 Peretto and Bonfanti. This is an open-access article distributed under the terms of the Creative Commons Attribution License (CC BY). The use, distribution or reproduction in other forums is permitted, provided the original author(s) or licensor are credited and that the original publication in this journal is cited, in accordance with accepted academic practice. No use, distribution or reproduction is permitted which does not comply with these terms. 